УДК 621.316.722.076.12; 504.05; 504

\title{
МНОГОФУНКЦИОНАЛЬНАЯ ОПТИМИЗАЦИЯ ПРИ КОМПЕНСАЦИИ РЕАКТИВНОЙ МОЩНОСТИ
}

Палау Илиана Антониа Гонсалес',

iliana.gonzalez@utc.edu.ec

\section{Рамирез Секундино Марреро',}

secundino.marrero@utc.edu.ec

\section{Балабанов Михаил Станиславович², balabanovms@mail.ru}

\section{Лобайна Аристидес Легра,} alegra@ismm.edu.cu

\author{
Мендиольа Даниель Мендиольа ${ }^{3}$, \\ dmendiola@ismm.edu.cu \\ 1 Технический университет Котопакси, \\ Эквадор, 050150, г. Котопакси, пр. Симона Родригеса, пр. Латакунга, б/н. \\ 2 Омский государственный технический университет, \\ Россия, 644050, г. Омск, пр. Мира, 11. \\ ${ }^{3}$ Высший металлургический институт Моа, им. доктора Антонио Нуньес Хименес, \\ Куба, 644050, г. Моа, Ольгин, пр. Каликсто Гарсия, 15.
}

\begin{abstract}
Актуальность. В настоящее время в России создается интеллектуальная энергосистема с активно-адаптивной сетью - ИЭС ААС (за рубежом - Smart Grid). Базовым кластером архитектуры Smart Grid являются FACTS-устройства, сложность проектирования которых заключается в многокритериальности задачи. Оптимизация реактивной мощности представляет собой подзадачу оптимального потока мощности, в ходе решения которой определяется правильная настройка переменных реактивной мощности, таких как величины: напряжения, положений ступеней трансформаторов и характеристик устройств компенсации реактивной мощности. Решение задач оптимизации реактивной мощности, которые не являются линейными и дискретными, при использовании традиционных методов оптимизации сопровождается определенными трудностями, связанными с обработкой данных разного характера. Поэтому в настоящее время ведется поиск адекватного способа многообъектной обработки данных, например, с помощью алгоритма эволюционной оптимизации.
\end{abstract}

Целью исследования является разработка математического метода поиска оптимального решения из всего множества возможных решений, которое было бы лучше других хотя бы для одной из поставленных целей. При этом модель должна выполнять расчет потока мощности на основной и гармонической частоте для конкретного режима, при большом количестве ограничений. Методы. Моделирование для внедрения устройств FACTS было выполнено в программе DYCSE. Использовался алгоритм случайного поиска, который является модификацией метода интеграции переменных. Он позволяет решать проблемы сходимости при применении к очень большому массиву данных. В качестве примера и основы для разработки обсуждаемого метода был принят метод расчета и результаты исследования Jose Arzola Ruiz. В данном исследовании оптимизации используется целевая функция метода Чебышева, позволяющая уменьшить взвешенное расстояние от расчетного до желаемого значения каждого индикатора, включенного в целевую функцию. Естественно, что популяция с высоким уровнем исходных данных представляет лучшее решение задачи и при определенных условиях может представлять даже единственно оптимальное решение. Из первоначального поколения потенциальных решений для процесса, который является повторяющимся, вычислялись новые поколения решений, каждый раз с лучшими характеристиками, наиболее близкими к оптимальному решению задачи. Критерии остановки расчета представляли собой смешанное условие - разницу между худшим и лучшим решениями. Каждый эксперимент выполнялся с начальной популяцией, которая имела случайный характер. Исследования показали, что при заданном диапазоне изменения любого параметра, применяемого для решения поставленной задачи компенсации реактивной мощности в электрических сетях, достаточно количество сочетаний ограничить 7 \% от всех возможных его значений. В результате реализации алгоритма получается множество решений с минимальными значениями целевой функции. В ходе расчета задача эксперта заключается в выборе рабочего варианта.

Результаты. Применение эволюционных методов в оптимизации позволяет одновременно рассматривать несколько независимых решений, создавая набор так называемых оптимально эффективных решений, или решений Pareto, которые удовлетворяют целям исследования. Во всех экспериментах были получены эффективные решения при оценке численности популяции около $10 \%$ от всех возможных решений. Полученные решения можно считать эффективными в сравнении с расчетами, которые можно было бы произвести при наличии абсолютно всех необходимых исходных данных и выполненных полномасштабных вычислений. Выводы. Для достижения энергоэффективности в промышленных сетях требуются новые методы оптимизации, позволяющие улучшить технико-экономические показатели сетей. Использование метода Чебышева позволяет уменьшить взвешенное расстояние от расчетного до желаемого значения каждого индикатора, включенного в целевую функцию. Теоретические расчеты прошли апробацию в ходе практических исследований. Доказано, что для значений, близких к 10 \% от спектра всех возможных решений, можно получить решения, которые удовлетворяют требованиям поиска эффективных решений. Разработанный алгоритм значительно сокращает время вычислений при гарантии сходимости результатов и совпадает с рекомендациями, предложенными Arzola.

\section{Ключевые слова:}

Многоцелевая оптимизация, компенсация реактивной мощности, FACTS-устройства, Smart Grid, эволюционные алгоритмы, генетические алгоритмы, расстояние Чебышева, гармоника. 


\section{Введение}

Компенсация реактивной мощности с математической точки зрения часто рассматривается как простая задача оптимизации с ограничениями. В этом случае поиск решения включает комбинации нескольких факторов, таких как потери электроэнергии при передаче (как следствие потери инвестиций), отклонения напряжения, снижение надежности. Оптимизация реактивной мощности представляет собой подзадачу оптимального потока мощности (при определенных ограничениях системы) [1-3], в ходе решения которой определяется правильная настройка переменных реактивной мощности, таких как величины напряжения, положения ступеней трансформаторов и характеристики устройств компенсации реактивной мощности.

В настоящее время решение проблем оптимизации реактивной мощности сфокусировано на традиционных методах оптимизации, включая метод градиента [4], квадратичного программирования [4], нелинейного/линейного программирования [5] и метод внутренней точки [4]. Кроме того, ведется поиск оптимальной многообъектной обработки данных с помощью алгоритма эволюционной оптимизации $[5,6]$. Решение задач оптимизации реактивной мощности, которые не являются линейными и дискретными, при обработке данных разного характера с использованием перечисленных методов сопровождаются определенными трудностями.

Генетические алгоритмы (ГА) (the Genetic Algorithms - GA) [4] являются наиболее используемым эвристическим методом в оптимизации реактивной мощности. Цель ГА - найти лучшее решение в спектре возможных. Под «лучшими решениями» мы понимаем те, которые позволяют получать значения, наиболее близкие к численному значению для конкретной функции $[7,8]$. Традиционные алгоритмы оптимизации позволяют получать приемлемое решение, однако применение эволюционных методов в оптимизации позволяет одновременно рассматривать несколько независимых решений, создавая набор так называемых оптимально эффективных решений, или решений Pareto [9], которые удовлетворяют целям исследования.

Трудности, возникающие в решениях, полученных с использованием метода оптимизации ГА, связаны с характером функциональной пригодности, формой кодирующих решений и разнообразием параметров. Чтобы преодолеть эти недостатки, были разработаны другие эвристические методы, которые поддерживают некоторые общие характеристики с генетическими алгоритмами, например, способ, продемонстрированный Jose Arzola Ruiz [4] на основе метода интеграций переменных (МИП) (the Method of Integration of Variables MIV). Это обобщение генетических алгоритмов, где параметры не обязательно обновляются с использованием кода для описания возможных ре- шений, но любой из них может быть пересчитан по указанию оператора.

Алгоритм случайного поиска (ACП) (the Conditional Random Search algorithm - CRS) [10] является модификацией МИП и позволяет решать проблемы сходимости при применении к очень большому массиву данных, в частности:

1) вести поиск решений подинтервалами (рис. 3,4$)$, увеличивать разнообразие исходной информации и улучшать качество решений во всем диапазоне;

2) вести поиск эффективных решений вблизи ранее рассчитанного решения для улучшения характеристик уже полученных решений;

3) вести поиск подходящих выборок решений на основе лучших характеристик уже рассчитанной совокупности, выбирать субпопуляцию, содержащую значения с благоприятными сегментами кода, которые могут значительно ускорить схождение метода.

\section{Компенсация реактивной энергии}

как многокритериальная система принятия решений

Описание компенсации реактивной мощности как процесса принятия решений приводит к внешнему анализу, который должен быть реализован на базе выбора координационных переменных, входных данных, технико-экономических показателей [2].

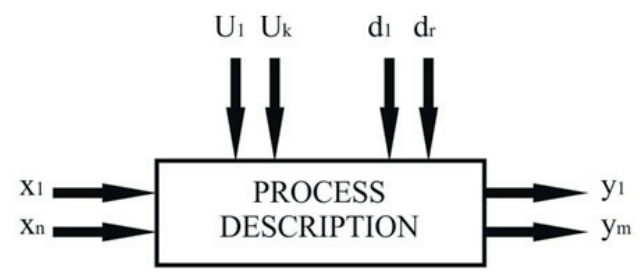

Рис. 1. Информационная классификация внешнего анализа

Fig. 1. Information classification of external analysis

На рис. 1 параметры $U_{1} \ldots U_{k}, d_{1} \ldots d_{r}$ - это независимые воздействия на систему. В качестве $U_{1} \ldots U_{k}$ принимаются данные по нагрузке в сети, а $d_{1} \ldots d_{r}-$ конфигурация схемы электроснабжения с параметрами кабельных и воздушных линий электропередачи.

$x_{1} \ldots x_{n}$ - параметры технических устройств, подлежащие изменению в процессе исследований. Например, параметры подключения: $x_{1}-$ трансформаторы; $x_{2}$ - конденсаторы; $x_{3}-$ фильтры; $x_{4}-$ синхронные двигатели с возбуждением и т. д.

$y_{1} \ldots y_{m}$ - технические и экономические показатели, которые могут характеризоваться как: $y_{1}-$ напряжение в узловых точках сети; $y_{2}-$ коэффициент мощности; $y_{3}$ - суммарные гармонические искажения; $y_{4}$ - общие потери энергии в сети; $y_{5}-$ экономическая переменная через чистую приведенную стоимость и т. д.

Чтобы получить необходимые индикаторы, нужны соответствующие методы моделирования. Характер используемого метода позволяет вклю- 
чать или даже исключать все технические и экономические показатели в соответствии с характеристиками сетей $[11,12]$.

В целом синтез системы для принятия решений включает в себя разработку или подбор необходимых инструментов для осуществления:

1) выбора технико-экономических показателей для заданных значений переменных решений;

2) построения вариантов решений, близких к компромиссу между выбранными индикатораМи;

3) построения графических параметров, которые позволяют увидеть особенности каждого варианта решения;

4) получения дополнительных опций моделирования, позволяющих более точно изучить особенности каждого генерируемого варианта.

Применение нетрадиционных методов оптимизации позволяет проводить многокритериальный анализ через объектную функцию (Fitnnes), не прибегая к использованию производных или градиентов в процессе поиска решений, близких к оптимальному, а также сокращает время обработки вычислений, не выполняя исчерпывающий поиск $[1,13-15]$.

\section{Математическая постановка задачи}

Как показано в работах $[16,12]$, для определения области эффективных решений можно воспользоваться методом Чебышева, который позволяет путем перебора минимизировать локальную целевую функцию по каждому критерию отдельно. Формально минимизация выглядит следующим образом.

$$
\max _{i}\left\{\omega_{i} \frac{\left|y_{c_{i}}-y_{d_{i}}\right|}{\left|y_{d_{i}}\right|}\right\},
$$

где $\omega_{i}$ - весовое соотношение, которое дает большее или меньшее значение между $i$-ми целями; $y_{c_{i}}-$ желаемые значения; $y_{d_{i}}$ - рассчитанные значения разных целей $y_{i}$.

Определив эффективное решение для одного показателя (используя уравнение 1), можно перейти к определению эффективных решений сразу для нескольких показателей на множестве различных комбинаций значений $\left(\omega_{i}\right)$, приводящих к созданию эффективного пространства решений. Учитывая преимущества, о которых говорилось ранее, предполагается в качестве частичной целевой функции (ЦФ) (the Objective Function - OF) на каждом узле $i=1 \mathrm{~m}$ системы воспользоваться следующим выражением

$$
Z_{i}=\max _{j}\left\{w_{i, j}\left|\frac{Z c_{i, j}-Z d_{i, j}}{Z d_{i, j}}\right|\right\},
$$

где $Z_{i}$ - значение частичной функции узла $i$ в диапазоне $0 \leq Z_{i} \leq 1 ; w_{i, j}-$ коэффициент веса в узле $i$ для каждого индикатора $j, 0 \leq w_{i, j} \leq 1 ; Z c_{i, j}, Z d_{i, j}$ - рассчитанное и желаемое значение в узле $i$ для объекта или индикатора $j$.
Весовые коэффициенты играют одну из важнейших ролей в выборе эффективных решений и должны отвечать следующим условиям: $0 \leq w_{i, j} \leq 1$; $\Sigma w_{i, j}=1$. Коэффициент $w_{i, j}$ может принимать разные значения в зависимости от анализируемой цели, но когда он принимает значение нуля, то он сводит на нет поиск.

В выражениях (1) и (2) $y_{c_{i}}$ и $Z d_{i, j}$, т. е. желаемые значения показателей, должны быть определены исходя из требований нормативных документов и рекомендаций, вводимых эксплуатирующей организацией. Значения этих ограничений используется в процессе поиска для каждой ступени итерационного расчета $[10,17]$. Тогда для каждого $i$-го узла и каждого $j$-го показателя целевая функция будет иметь вид:

$$
Z_{i j}=w_{i j}\left|\frac{V c_{i j}-V d_{i j}}{V d_{i j}}\right|,
$$

Значение $Z_{i j}$ всегда будет от 0 до 1 .

Для случая трех показателей в узлах электрической сети целевая функция примет вид:

$$
Z_{I J}=\lambda_{1} Z_{I 1}+\lambda_{2} Z_{I 2}+\lambda_{3} Z_{i 3},
$$

где $\lambda$ - коэффициент веса для $i$-го узла; $Z_{I}$ - результат $Z$ в узле для объективного $(i)$ с наихудшим значением. Уравнение включает в себя только те цели, которые будут проанализированы на уровне узла: $Z_{I 1}$ (напряжение), $Z_{I 2}$ (коэффициент мощности) и $Z_{\text {I3 }}$ (гармонические искажения напряжения).

После определения, в зависимости от заданного ограничения, значений $Z_{I J}$ формируется обобщенная целевая функция, включающая дополнительные составляющие, характеризующие работу всей сети целиком. Допустим, такими показателями являются суммарные потери мощности в сети и экономический показатель, которым соответствуют локальные целевые функции $Z_{4}$ и $Z_{5}$. Тогда обобщенная целевая функция будет иметь вид:

$$
Z_{T}=\lambda_{1} Z_{1}+\lambda_{2} Z_{2}+\lambda_{3} Z_{3}+\lambda_{4} Z_{4}+\lambda_{5} Z_{5},
$$

где $\lambda_{1}-\lambda_{5}-$ константа веса для узлов $i$ (диапазон от 0 до 1) соответствует типу узла и индикаторам, которые будут зависеть от важности каждого конкретного случая. Как объяснялось ранее, все значения регулируются между 0 и 1 . Сумма весовых коэффициентов должна быть равна 1. Например, если необходимо выделить только экономический показатель, то в этом случае он будет учтен со значением, равным 1.

Математическая модель выполняет расчет потока нагрузки на основной и гармонической частоте, для конкретного исследования. Чтобы обеспечить приемлемый сектор решений, необходимо принять во внимание перечень ограничений, которые даются:

1) на ёмкостную реактивную мощность узла, которая должна находиться в допустимых пределах;

2) коэффициент мощности вводного узла;

3) коэффициент мощности узлов потребителей;

4) чистую приведенную стоимость (ЧПС). 


\section{Внедрение метода оптимизации}

Метод переленных интеграций [9]. Структура популяции определяется набором переменных решений, участвующих в процессе оптимизации. Для получения возможных решений существует механизм кодирования, который позволяет присваивать каждому отдельному параметру единое значение, которое отражает его качество как решение.

Естественно, что популяция с высоким уровнем исходных данных представляет лучшее решение проблемы и при определенных условиях может представлять даже единственно оптимальное решение $[18,19]$.

Из первоначального поколения потенциальных решений для процесса, который является повторяющимся, выполняются новые поколения решений с лучшими характеристиками, которые каждый раз приближают задачу к оптимальному ее решению [20].

Выбор критериев окончания расчета, в общем случае, обусловлен особенностями каждого конкретного приложения. Во внимание принимаются следующие критерии:

1) количество итераций без изменений в популяции;

2) разница между лучшим и худшим решением, уступающим заранее установленному значению в популяции;

3) предопределенное количество вариантов сгенерированных решений;

4) смешанные условия.

Алгоритм МПИ показан на рис. 2.

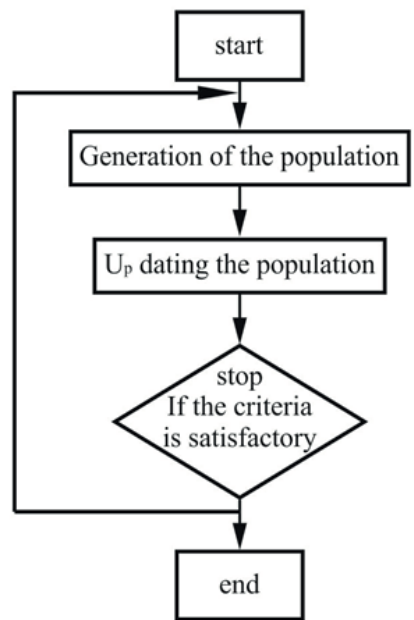

Рис. 2. Алгоритм метода интеграции переменных

Fig. 2. Algorithm of variables integration method

Метод случайного условного поиска (МСУП) (the Random Conditional Search - RCS) следует основным идеям метода МИП, предложенного Arzola [4] (рис. 2). Этот метод состоит из серии алгоритмов, с помощью которых осуществляется поиск начальной популяции, улучшение начальной популяции и выборочное сокращение популяции.
Алгоритл поиска первоначальной совокупности (ПС) (the Initial Population - IP) состоит из множества $K$ элементов, назначенных исследователем [21]. Для каждой итерации расчета наилучшее решение включено в популяцию, и эти расчеты повторяются до тех пор, пока размер популяции не совпадает с первоначальным.

Существует два шага получения ПС, предполагая, что $T$ представляет собой совокупность всех возможных решений системы:

1) возьмите $k$ случайных чисел между 1 и $T$;

2) разделите интервал от 1 до $T$ на $K$ подинтервалов и получите решение ПС в каждом промежуточном интервале.

Первоначальное улучшение численности популяиии. После того как ПС имеет фиксированное значение, производится его обновление, путем сравнения полученных решений в целевой функции. Выбирается лучшее из решений, то есть то, которое имеет меньшее значение $Z$, и это новое решение сравнивается с уже рассчитанным наихудшим решением популяции. Определяется возможность замены. В тот момент, когда достигается точность $\delta$, процесс генерации случайных значений популяции перезапускается.

Для каждой итерации генерируются два кода переменных в интервале [A,B] (рис. 3). Между А и В три подинтервала $\left[\mathrm{A}, \mathrm{X}_{1}\right],\left[\mathrm{X}_{1}, \mathrm{X}_{2}\right]$ и $\left[\mathrm{X}_{2}, \mathrm{~B}\right]$. Исключается интервал, содержащий более высокое значение $Z$, что является наихудшим результатом $\left[\mathrm{X}_{2}, \mathrm{~B}\right]$ (рис. 3). Затем в пределах полученного интервала $\left[\mathrm{A}, \mathrm{X}_{2}\right]$ генерируются два других случайных значения. Этот процесс повторяется до тех пор, пока на каждую стадию добавляется решение с более низким значением $Z$. При вычислении меньшего значения $Z$ оно заменяет наихудшее решение, которое уже было найдено на предыдущих этапах.

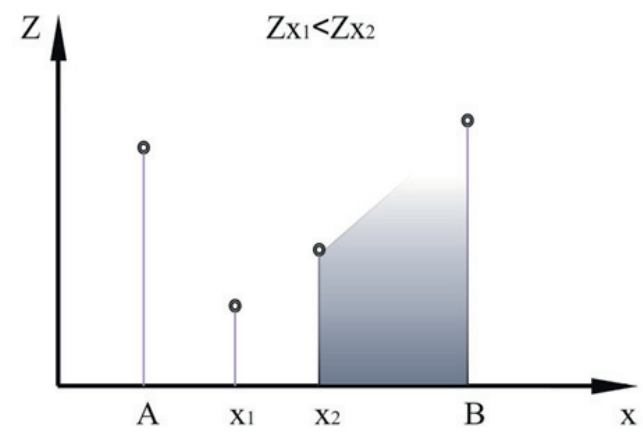

Рис. 3. Первый шаг для начального поиска популяции в интервале $[A, B]$, исключая интервал $\left[X_{2}, B\right]$

Fig. 3. First step for population initial search in the interval $[A, B]$, eliminating the interval $\left[X_{2}, B\right]$

Существенным моментом при реализации представленного алгоритма является определение количества изменения параметра $X_{n}$ при достижении значения $Z_{\max }$. Для разветвленной электрической сети решение на основе метода Чебышева может содержать сотни тысяч вариантов решений. 
Уменьшить количество расчетных точек и сократить время расчета позволяет метаэвристический метод. Алгоритм нацелен на поиск решений с уменьшающимися значениями $Z$, что означает сходимость алгоритма. Для исключения возможности появления значений меньших, чем рассчитанный $Z_{\text {х1 }}$, были реализованы возможности усечения в основном алгоритме [22].

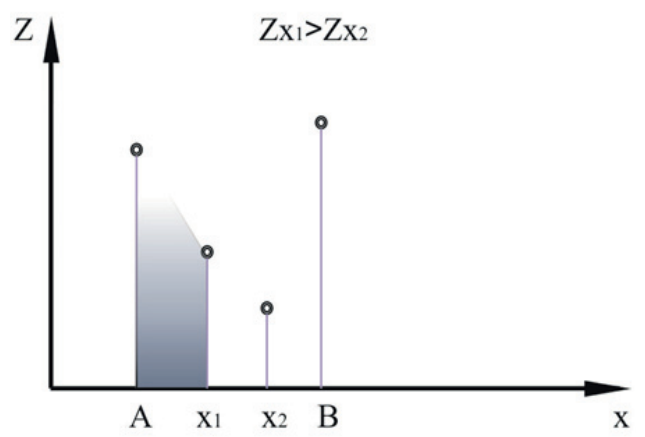

Рис. 4. Второй шаг для начального поиска популяций в $\left[A, X_{1}\right]$

Fig. 4. Second step for population initial searching in the $\left[A, X_{1}\right]$

Исследования показали, что при заданном диапазоне изменения любого параметра, применяемого для решения поставленной задачи компенсации реактивной мощности в электрических сетях, достаточно его изменение ограничить $7 \%$ от всех возможных его значений. Такое утверждение следует из анализа зависимости, представленной на рис. 5. График был получен из нескольких экспериментов, в которых отражается конвергенция (прямая линия от 7 \% расчета всей совокупности и далее), поэтому делается вывод, что из $10 \%$ можно гарантированно получить хорошие решения или решения, близкие к оптимальным.

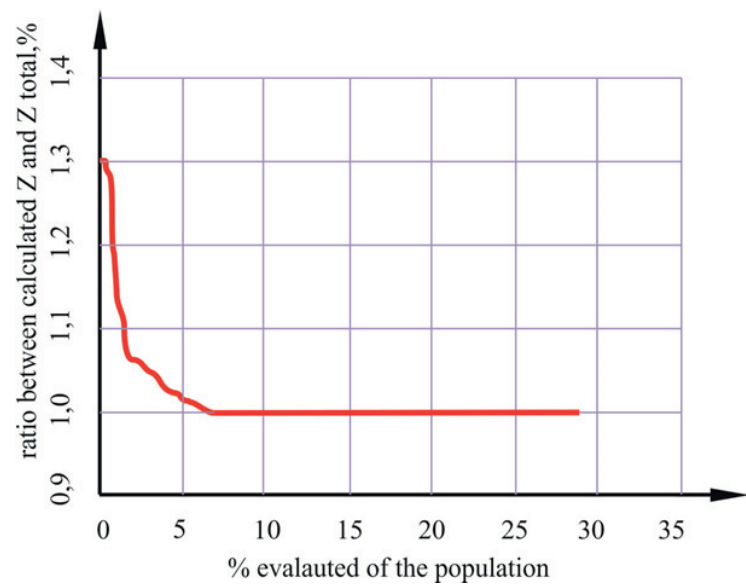

Рис. 5. Среднее соотношение рассчитанных Z и процентов от общей популяции значений

Fig. 5. Average ratio of calculated $Z$ and percent of population evaluated

\section{Кодирование переменных полученных решений}

Переменные решения могут принимать разные конфигурации, определяемые как $(x)$. Исследова- ние предполагало, что $(x)$ является строковым символом, где каждый символ представляет собой трансформатор, батарею конденсаторов или фильтр в схеме, и каждое возможное значение $(x)$ зависит от определения анализируемого элемента.

Например, электрическая схема состоит из: трансформатора $(T)$ с двумя выводами 1 и 2 ; конденсаторной батареи $(C)$ с двумя переключаемыми секциями 1 и 2 ; фильтра $(F)$ с двумя состояниями (включен или выключен). Тогда число независимых комбинаций (ЧНК) конфигураций, принятых этими переменными решениями, было бы: ЧНК $=2 * 3 * 2=12$.

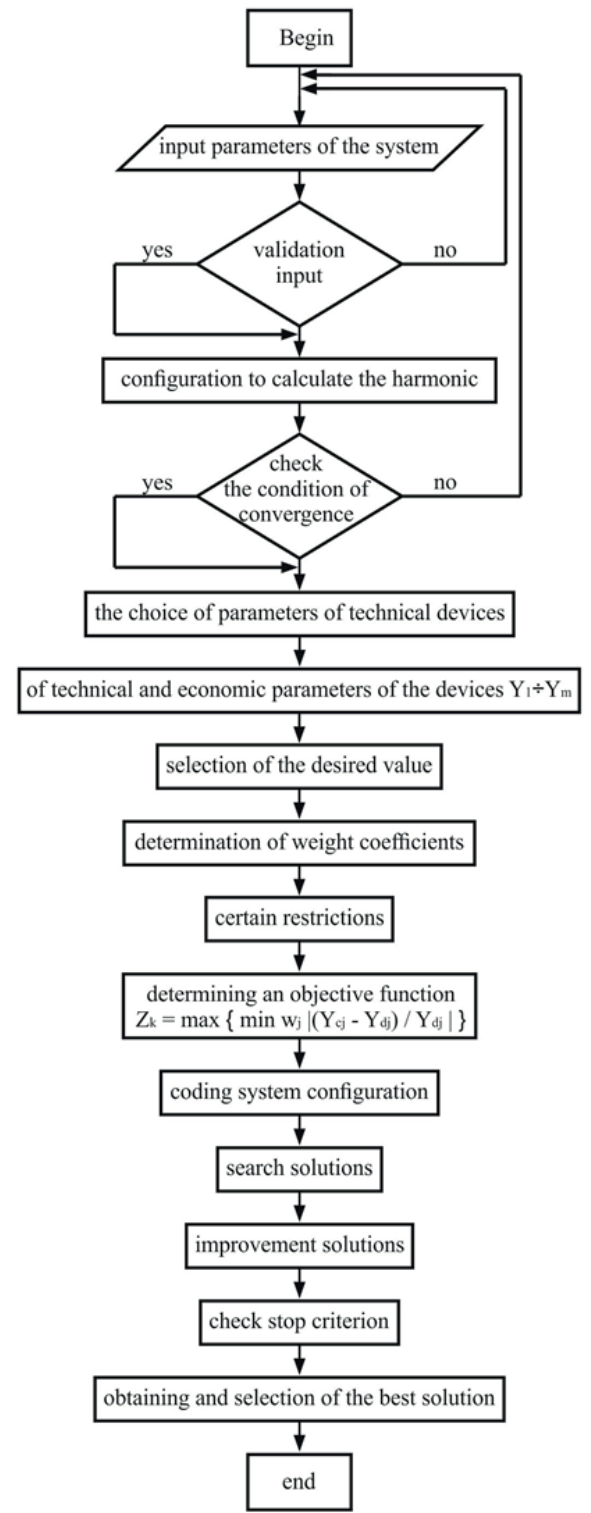

Рис. 6. Общий алгоритм достижения эффективных решений

Fig. 6. General algorithm for achieving efficient solutions

Необходимо отметить, что предлагаемое кодирование является десятичным, то есть каждая цифра кода принимает десятичные значения. Следовательно, необходим специальный алгоритм для 
реализации кодирования в двух функциях, которые представляют значения $X$. C помощью этих двух функций получается код $(X)$, гарантирующий однозначным образом кодификацию для каждого элемента электрической системы - трансформаторов, фильтров и банок конденсаторов.

Критерии остановки и проверки алгоритла оптилизации. Критерии остановки, используемые при оптимизации, представляют собой смешанное условие, в котором вычисляется ряд кодов решений без изменений в составе популяции. Разница между вычисленными значениями $Z$ в целевой функции должна быть меньше заданного значения (худшее и лучшее решение) [23].

Каждый эксперимент выполняется с начальной популяцией, которая имеет случайный характер, получая приемлемые решения из 7 \% оцениваемых (рис. 5,6 ) [9]. Из чего можно сделать вывод, что для значений, близких к 10 \% от спектра всех возможных решений, можно получить решения, которые гарантируют выполнение требований поиска эффективных решений, совпадающие с рекомендациями, предложенными Arzola [4]. В этих экспериментах стандарт качества, используемый для оценки решений, соответствует наилучшему решению, найденному в исчерпывающем поиске, представленном через $Z_{\text {еха }}$.

Результаты экспериментов по проверке сходимости алгоритма, показанного на рис. 5, были получены для системы, показанной на рис. 8. Системные данные для моделирования и полученные эффективные решения приведены в табл. 1,2 соответственно.

С другой стороны, для больших систем поставленная задача представляет собой проблему: если одновременно оценивать разные индикаторы, то можно получить сомнительные решения. Поэтому удобно использовать эволюционные методы, которые позволяют получать решения, близкие к эффективным значениям, а следовательно позволяют оценить во всей сложности такую систему, как электрическая схема [24].

\section{Проверка сходимости}

В табл. 1 показана активная и реактивная мощность, согласно схеме на рис. 7.

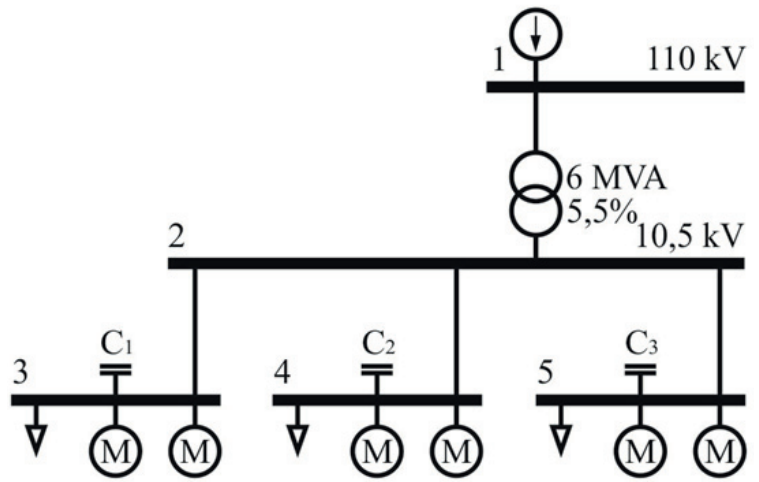

Рис. 7. Энергетическая система для оценки сходимости метода

Fig. 7. Power system to evaluate the convergence of the method
Таблица 1. $Q_{c}$ и нагрузки, установленные в системе на рис. 8 до оптимизации

Table 1. $Q_{c}$ and loads installed in the system of Fig. 8 before optimization

\begin{tabular}{|c|c|c|c|}
\hline $\begin{array}{c}\text { Узлы } \\
\text { Nodes }\end{array}$ & $\begin{array}{c}\text { Активная } \\
\text { мощность, кBт } \\
\text { Active power, } \\
\mathrm{kW}\end{array}$ & $\begin{array}{c}\text { Реактивная } \\
\text { мощность, кBAp } \\
\text { Reactive power, } \\
\mathrm{kVAr}\end{array}$ & $\begin{array}{c}\text { Емкостная } \\
\text { мощность, } Q_{c}, \mathrm{KBAp} \\
\text { Capacitive Power, } Q_{c} \\
\mathrm{kVAr}\end{array}$ \\
\hline 3 & 1053 & 390 & 450 \\
\hline 4 & 81 & 34 & 34 \\
\hline 5 & 2050 & 874 & 460 \\
\hline
\end{tabular}

Результаты поиска эффективных решений представлены в табл. 2. Как видно, значение $Z$ является минимальным в варианте № 1, что является лучшим решением с точки зрения потерь энергии. Результаты расчетов по известным методам и методу, разработанному авторами, совпали. Проверка сходимости результатов успешно пройдена [25].

Таблица 2. Значения $\cos \varphi$ и потери для трех эффективных решений, найденных при оптимизации

Table 2. Values of $\cos \varphi$ and losses for three of the efficient solutions found during optimization

\begin{tabular}{|c|c|c|c|c|c|c|}
\hline Bычисленные значения Z & \multicolumn{3}{|c|}{$\cos \varphi$} & \multicolumn{3}{c|}{$Q_{c}$ (кBAp/kVAr) } \\
\cline { 2 - 7 } Calculated values $Z$ & 4 & 5 & 6 & $C_{1}$ & $C_{2}$ & $C_{3}$ \\
\hline $0,736 \cdot 10^{-2}$ & 0,96 & 0,94 & 0,94 & 472 & 27 & 375 \\
\hline $0,762 \cdot 10^{-2}$ & 0,95 & 0,95 & 0,94 & 450 & 34 & 375 \\
\hline $0,771 \cdot 10^{-2}$ & 0,95 & 0,95 & 0,95 & 450 & 34 & 469 \\
\hline $0,923 \cdot 10^{-2}$ & 0,93 & 0,92 & 0,93 & 225 & 17 & 234 \\
\hline
\end{tabular}

\section{Пример исследования}

На рис. 8 показана модель предлагаемого алгоритма МСУП.

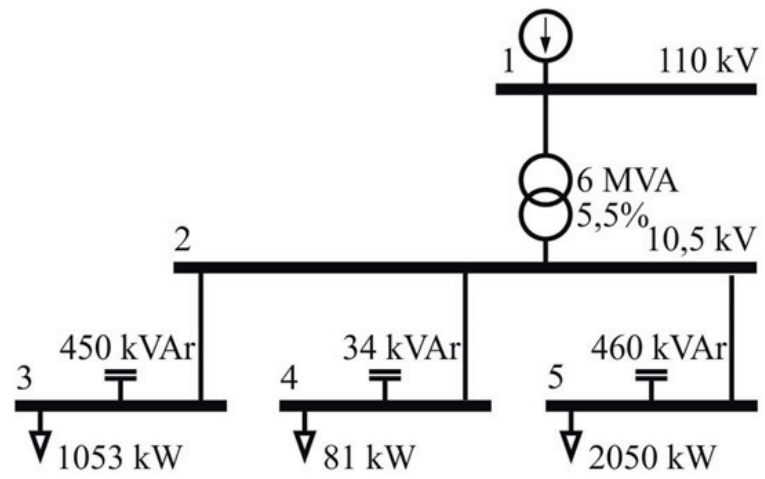

Рис. 8. Электрическая схема

Fig. 8. Electric diagram

В табл. 3, 4 представлены результаты трех решений, согласно рис. 8 , где решение 1 имеет наибольшее значение, решение 2 - среднее значение, а решение 3 - наименьшее значение.

Изменение коэффициента мощности при включении различных ступеней конденсаторных установок представлено в табл. 2.

В табл. 5, 6 показаны четыре оптимальных решения (так называемые решения Pareto) из расче- 
та от 7 до $10 \%$ от общей численности популяции. Предложенный алгоритм значительно сокращает время вычислений при гарантии сходимости.

Таблица 3. Значение потерь и $\cos \varphi$ для разных решений Table 3. Value of losses and $\cos \varphi$ for different solutions

\begin{tabular}{|c|c|c|c|c|c|}
\hline \multirow{3}{*}{\multicolumn{2}{|c|}{$\begin{array}{l}\text { Расчетное значение ( } Z \text { ) } \\
\text { в целевой функции } \\
\text { Calculated value of ( } Z \text { ) } \\
\text { in the objective function }\end{array}$}} & \multirow{3}{*}{$\begin{array}{c}\Delta E \\
(\mathrm{KBT} 4) \\
(\mathrm{kWh})\end{array}$} & \multirow{2}{*}{\multicolumn{3}{|c|}{$\frac{\cos \varphi}{\text { зел/Node }}$}} \\
\hline & & & & & \\
\hline & & & 3 & 4 & 5 \\
\hline Решение/Solution & \multirow{2}{*}{0,025} & \multirow{2}{*}{298015} & \multirow{2}{*}{0,90} & \multirow{2}{*}{0,76} & \multirow{2}{*}{0,92} \\
\hline 1 & & & & & \\
\hline 2 & 0,019 & 283824 & 0,94 & 0,89 & 0,92 \\
\hline 3 & 0,009 & 276728 & 0,93 & 0,92 & 0,93 \\
\hline
\end{tabular}

таблица 4. Положение ступени трансформатора и Q

Table 4. Transformers tap position and $Q_{c}$

\begin{tabular}{|c|c|c|c|c|c|}
\hline \multirow{2}{*}{$\begin{array}{c}\text { Расчетное значение (Z) } \\
\text { в целевой функции } \\
\text { Calculated value of ( Z) } \\
\text { in the objective function }\end{array}$} & \multicolumn{4}{|c|}{$\begin{array}{c}\text { Ступень трансформатора } \\
\text { и значение } Q_{c,} \text { кBAp }\end{array}$} \\
\cline { 2 - 6 } & \multicolumn{4}{|c|}{$\begin{array}{r}\text { Transformer position and value } \\
\text { of } Q_{c} \text { in kVAr }\end{array}$} \\
\hline $\begin{array}{c}\text { точка 1 } \\
\text { роint 1 }\end{array}$ & 0,025 & 2 & 472 & 17 & 234 \\
\hline $\begin{array}{c}\text { точка 2 } \\
\text { роint 2 }\end{array}$ & 0,019 & 3 & 472 & 17 & 469 \\
\hline $\begin{array}{c}\text { точка 3 } \\
\text { роint 3 }\end{array}$ & 0,009 & 6 & 225 & 17 & 234 \\
\hline
\end{tabular}

\section{Выводы}

Уменьшение гармонических искажений и компенсацию реактивной мощности можно сформулировать как задачу принятия решений по нескольким критериям в дискретных переменных с различными связанными с ними техническими и экономическими показателями. Эффективность решений достигается использованием значений Чебышева целевой функции.

Характер сформулированной задачи для компенсации реактивной мощности позволяет использовать процедуры для генерации решений, основанных на эволюции кодов, то есть любой из соответствующих алгоритмов метода интеграции переменных. Алгоритм условного случайного поиска

\section{СПИСОК ЛИТЕРАТУРЫ}

1. Optimal power flow solutions using differential evolution algorithm integrated with effective constraint handling techniques / P.P. Biswas, P.N. Suganthana, R. Mallipeddi, G.A.J. Amaratunga // Engineering Applications of Artificial Intelligence. - February 2018. - V. 68. - P. 81-100. DOI: https://doi.org/10.1016/j.engappai.2017.10.019

2. Optimal reconfiguration and capacitor placement for power loss reduction of distribution system using improved binary particle swarm optimization / M. Sedighizade, M. Dakhem, M. Sarvi, H.H. Kordkheili // International Journal of Energy and Environmental Engineering. - April 2014. - V. 5. - № 1. - P. 1-11. DOI $10.1007 /$ s40095-014-0073-9

3. A controlled device for reactive-power compensation for electrified alternating-current / A.N. Marikin, V.A. Miroshchenko, быстро и эффективно генерирует ряд коррелированных решений с задачей оптимизации, а также облегчает оценку нескольких корректирующих элементов вариантов подключения в сети, чтобы облегчить работу по принятию решения.

Таблица 5. Расчетные значения четырех выбранных оптимальных решений

Table 5. Calculated values of four of the selected optimal solutions

\begin{tabular}{|c|c|c|c|c|c|}
\hline \multirow{2}{*}{$\begin{array}{l}\text { Решение } \\
\text { Solution }\end{array}$} & \multirow{2}{*}{$\begin{array}{c}\text { Расчетные значения } \\
(Z) \\
\text { Calculated values of }(Z)\end{array}$} & \multirow{2}{*}{$\begin{array}{c}\Delta E \\
(\mathrm{kBT \varphi}) \\
(\mathrm{kWh})\end{array}$} & \multicolumn{3}{|c|}{$\begin{array}{l}\cos \varphi \text { в узлах нагрузки } \\
\cos \varphi \text { in the load nodes }\end{array}$} \\
\hline & & & 4 & 5 & 6 \\
\hline 1 & 0,007 & 272471 & 0,96 & 0,94 & 0,94 \\
\hline 2 & 0,007 & 274599 & 0,95 & 0,95 & 0,94 \\
\hline 3 & 0,007 & 270342 & 0,95 & 0,95 & 0,95 \\
\hline 4 & 0,009 & 276728 & 0,93 & 0,92 & 0,93 \\
\hline
\end{tabular}

Таблица 6. Изменение ступени трансформаторов и мощности конденсаторов для оптимальных решений

Table 6. Change in transformer taps and capacitive reactive power for optimal solutions

\begin{tabular}{|c|c|c|c|c|}
\hline \multirow{2}{*}{$\begin{array}{c}\text { Peшение } \\
\text { Solution }\end{array}$} & $\begin{array}{c}\text { Супень трансформатора } \\
\text { Transformer taps }\end{array}$ & \multicolumn{3}{|c|}{$\begin{array}{l}Q_{c} \text { в конденсаторах, } \mathrm{kBAp} \\
Q_{c} \text { in the capacitors in kVAr }\end{array}$} \\
\cline { 2 - 5 } & $\mathrm{T1}$ & $\mathrm{C} 1$ & $\mathrm{C} 2$ & $\mathrm{C} 3$ \\
\hline 1 & 6 & 472 & 27 & 375 \\
\hline 2 & 6 & 450 & 34 & 375 \\
\hline 3 & 6 & 450 & 34 & 469 \\
\hline 4 & 6 & 225 & 17 & 234 \\
\hline
\end{tabular}

Для лучшего выбора зарезервированных индикаторов можно использовать значения в целевой функции. В случае большой размерности системы значения должны определяться с помощью экспериментальных данных (учитывая значимость анализируемого индикатора) чтобы уменьшить количество коэффициентов значений объективной функции.

Во всех экспериментах были получены эффективные решения при оценке поиска первоначальной совокупности около 10 \% от всех возможных решений. Полученные решения можно считать эффективными в сравнении с расчетами, когда известны абсолютно все необходимые исходные данные и выполнены полномасштабные расчеты.

V.V. Nikitin, A.V. Tret'yakov // Russian Electrical Engineering. October 2017. - V. 88. - № 10. - P. 639-642. DOI: https://doi.org/10.3103/S1068371217100091

4. Jose A.R. Sistemas de ingenieria. - La Habana: Editorial Félix Varela, 2000. - $482 \mathrm{p}$.

5. Iliana G.P. Optimization of reactive power compensation in industrial power supply networks using a multi criteria algorithm // Energy. - 2006. - V. XXVII. - № 2. - P. 40-44.

6. Alejandro C., Demetrio R.L., Juan M. Metaheuristic optimization applied to the calculation of optimum load flow in electric power grids with mixed type control variables // Engineering Journal UC. - December 2013. - V. 20. - № 3. - P. 71-78. D0I: $\mathrm{http} /$ www.redalyc.org/articulo.oa?id:70732641009

7. Iliana G.P., Secundino M.R. Reactive power compensation by evolutionary techniques // The VII International Conference for the 
Exploitation of Mineral Resources «CINAREM 2013». - Moa, province of Holguín, Cuba, November 19-21, 2013. - P. 1-7.

8. Yun Wei Li, Jinwei He. Distribution system harmonic compensation methods: an Overview of DG-Interfacing inverters // IEEE. Industrial Electronics Magazine. - November 2014. - № 8. Iss. 4. - P. 18-31. DOI: 10.1109/MIE.2013.2295421

9. Шклярский Я.Э., Гонсалес Палау И. Оптимизация компенсации реактивной мощности в сложных электрических сетях // Записки Горного института. - 2011. - Т. 194. - С. 349-352.

10. Metaheuristics based on the variables integration method applied to reactive power compensation in multi-objective optimization / G.P. Iliana, M.R. Secundino, L.L. Aristides, M.E. Daniel // Proc. of the International Conference on Applied Mathematics, Computational Science \& Engineering (AMCSE 2015). - Crete, Greece, October 17-19 2015. - P. 207-212.

11. Гонсалес Палау И. Выбор параметров экономически выгодной компенсации реактивной мощности // Записки Горного института. - 2011. - Т. 194. - С. 235-239.

12. Utopian point based decomposition for multi-objective optimization problems with complicated Pareto fronts / Yutao Qi, Qingsai Zhanga, Xiaoliang Ma, Yining Quana, Qiguang Miaoa // Applied Soft Computing. - December 2017. - V. 61. - P. 844-859. D0I: https://doi.org/10.1016/j.asoc.2017.08.036

13. Abd-Elazim S.M., Ali E.S. Synergy of particle swarm optimization and bacterial foraging for TCSC damping controller design // Journal of WSEAS Transactions on Power systems. - April 2013. - V. 8. - № 2. - P. 74-84.

14. Ali E.S., Abd-Elazim S.M. Power system stability enhancement via bacteria foraging optimization algorithm // Arabian journal for Science and Engineering (AJSE). - March 2013. - V. 38. № 3. - P. 599-611. DOI: 10.1007/s13369-012-0423-y

15. Ali E.S., Abd-Elazim S.M. Optimal PSS design in a multimachine power system via bacteria foraging optimization algorithm // Journal of WSEAS Transactions on Power systems. - October 2013. - V. 8. - № 4. - P. 186-196.

16. Applying distances between terms to both at and hierarchical data / J.A. Bedoya-Puerta, J. Hernandez-Orallo, C. Ferri, M.-J. Ramrez-Quintana // Proc. of AAIP 2011. $4^{\text {th }}$ International Workshop on Approaches and Applications of Inductive Programming. Denmark, July 2011. - P. 1-16.

17. Modeling of electric network regimes in the presence of harmonic distortion / P.I. Gonzalez, R.S. Marrero, L.A. Legra, S.A. Leon,
M.X. Proano // Energy Technical Magazine. - 2017. - № 13. P. $97-105$.

18. Barrico C., Antunes C.H., Pires D.F. Robustness analysis in evolutionary multi-objective optimization applied to VAR planning in electrical distribution networks // Evolutionary Computation in Combinatorial Optimization. EvoCOP 2009. Lecture Notes in Computer Science / Eds. C. Cotta, P. Cowling. - Berlin, Heidelberg: Springer, 2009. - V. 5482. - P. 216-227. DOI: https://doi.org/10.1007/978-3-642-01009-5_19

19. Muthukumar K., Jayalalitha S. Multiobjective hybrid evolutionary approach for optimal planning of shunt capacitors in radial distribution systems with load models // Ain Shams Eng J. 2017. - P. 1-14. DOI: http://dx.doi.org/10.1016/j.asej.2017.02.002

20. Vasconcellos D.B., Abril I.P., Martínez V.L. Reactive power compensation in unbalanced systems using genetic algorithms // Ingeniare. Chilean journal Engineering. - 2012. - V. 20. - № 3. - P. 284-292. DOI: http://dx.doi.org/10.4067/S0718-33052012000300002.

21. Secundino M.R., Iliana G.P., Arístides L.L. Convergence analysis of the variables integration method applied in multiobjetive optimization in the reactive power compensation in the electric nets // DYNA 82 (190). - April 2015. - P. 160-165. DOI: http://dx.doi.org/10.15446/dyna.v82n190.43697

22. Sharaf A.M., Elgammal A. A novel discrete multiobjective particle swarm optimization (MOPSO) of optimal shunt power filter // IEEE/PES Power Systems Conference and Exposition. 2009. P. 1-7. DOI: 10.1109/PSCE.2009.4839957

23. Schweickardt G.A. Multiobjective metaheuristic FPSO- $\chi$. An application to mid/long term expansion planning of an electric distribution system // Energetica. - 2009. - № 42. - P. 73-88. DOI: $10.15446 /$ energética

24. Rueda V.M., Velazquez J.D., Franco C.J. Recent advances in load forecasting using nonlinear models // DYNA. - Medellin, Junio, 2011. - № 78 (167). - P. 36-43.

25. Shklyarskiy E., Skamyin A.N. Compensation of the reactive power in the presence of higher voltage harmonics at coke plants // Coke and Chemistry. - 2016. - V. 59. - № 4. - P. 163-168. DOI: https://doi.org/10.3103/S1068364X16040062

Поступила 22.12.2017 г.

\section{Информация об авторах}

Палау Илиана Антониа Гонсалес, кандидат технических наук, профессор Технического университета Котопакси.

Рамирез Секундино Марреро, кандидат технических наук, декан кафедры электротехники Технического университета Котопакси.

Балабанов M.С., аспирант кафедры электрической техники Омского государственного технического университета.

Лобайна Аристидес Легра, кандидат технических наук, доцент кафедры математики Высшего металлургического института Моа, им. доктора Антонио Нуньес Хименес.

Мендиольа Даниель Мендиольа, кандидат технических наук, доцент кафедры электротехники Высшего металлургического института Моа, им. доктора Антонио Нуньес Хименес. 


\title{
MULTIOBJECTIVE OPTIMIZATION IN REACTIVE POWER COMPENSATION
}

\author{
Iliana Antonia G. Palau', \\ iliana.gonzalez@utc.edu.ec
}

\section{Secundino M. Ramirez',}

secundino.marrero@utc.edu.ec

\section{Mikhail S. Balabanov²,}

balabanovms@mail.ru

\author{
Aristides L. Lobaina ${ }^{3}$, \\ alegra@ismm.edu.cu \\ Daniel M. Ellis ${ }^{3}$, \\ dmendiola@ismm.edu.cu \\ ${ }^{1}$ Cotopaxi Technical University, \\ S/N, Simón Rodriguez Avenue, Latacunga, Cotopaxi, 050150, Ecuador. \\ 2 Omsk State Technical University, \\ 11, Mira Avenue, Omsk, 644050, Russia. \\ ${ }^{3}$ Higher Metallurgical Mining Institute of Moa, Dr. Antonio Nunez Jimenez, \\ 15, Calixto Garcia Avenue, Moa, Holguin, 83310, Cuba.
}

Relevance. Currently, Russia is creating an intelligent power system with an actively-adaptive network - IES AAS (abroad - Smart Grid). The basic Smart Grid group architecture is FACTS-devices; the complexity is the multi-criterion nature of the problem. Reactive power optimization is a secondary problem of the optimal power flow, when the setting of the correct reactive power variables, such as values of voltage, transformer position stages and reactive power characteristics of compensation devices, is determined. The solution for the problems to reactive power optimization, which are not linear and discrete, using traditional optimization methods is accompanied by certain difficulties associated with the processing of data that has different nature. Therefore, at the present, an adequate method of multi-object data processing is being searched, for example, using the evolutionary optimization algorithm

The aim of the research is to develop a mathematical method to find an optimal solution from the whole set of possible ones, which would be better than others for at least one objective. In this case, the model must perform a calculation of the power flux at the fundamental and harmonic frequency for a particular mode, with a large number of restrictions.

Methods. The simulation modeling of the FACTS device implementation was carried out in a program called DYCSE. We used the random search algorithm, which is a variable integration method modification and allows solving convergence problems when it is applied to a very large data set. The calculation method and the results of the study of Arzola Ruiz Jose As were taken as an example and the basis for developing our method. The Chebyshev method was used in the objective function. This methods allows reducing the weighted distance from the calculated value to the desired one of each indicator included in the objective function. It is obvious that a population with a high level of initial data represents the best solution to the problem and, under certain conditions, it can present even the only optimal solution. From the initial generation of potential solutions for the process that is repetitive, the new generations of solutions were derived, each time with better characteristics approaching the optimal solution of the problem. The criteria for stopping the calculation were a mixed condition - the difference between the worst and the best decisions. Each experiment was performed with the initial population that has a random character, obtaining acceptable solutions about $7 \%$ according to the estimated value.

Results. The use of evolutionary methods in optimization allows simultaneous consideration of several independent solutions, creating a set of so-called optimally effective solution or Pareto solutions that satisfy the research objectives. In all the experiments, the effective solutions were obtained to estimate the population size about $10 \%$ of all possible solutions. The obtained solutions can be considered effective in comparison with the calculations that could be performed with absolutely all required initial data and full-scale calculations performed.

Conclusions. To achieve energy efficiency in industrial networks, new optimization methods that improve the technical and economic performance of networks are required. The use of the Chebyshev method, which makes it possible to reduce the weighted distance from the calculated value to the desired one of each indicator included in the objective function, was tested obtaining satisfactory results in theoretical and practical studies. It is proved that for values close to $10 \%$ of the spectrum of all possible solutions we can obtain solutions that satisfy the search for effective solutions, and that coincide with the recommendations proposed by Arzola. The developed algorithm significantly reduces the computation time, with results convergence guarantee.

Key words:

Multi-objective optimization, reactive power compensation, FACTS-devices, Smart Grids, evolutionary algorithms, genetic algorithms, Chebycheff distance, harmonic. 


\section{REFERENCES}

1. Biswas P.P., Suganthana P.N., Mallipeddi R., Amaratunga G.A.J. Optimal power flow solutions using differential evolution algorithm integrated with effective constraint handling techniques. Engineering Applications of Artificial Intelligence, February 2018, vol. 68, pp. 81-100. D0I: https://doi.org/ 10.1016/j.engappai.2017.10.019

2. Sedighizade M., Dakhem M., Sarvi M., Kordkheili H.H. Optimal reconfiguration and capacitor placement for power loss reduction of distribution system using improved binary particle swarm optimization. International Journal of Energy and Environmental Engineering, April 2014, vol. 5, no. 1, pp. 1-11. DOI: 10.1007/s40095-014-0073-9

3. Marikin A.N., Miroshchenko V.A., Nikitin V.V., Tret'yakov A.V. A controlled device for reactive-power compensation for electrified alternating-current. Russian Electrical Engineering, October 2017, vol. 88, no. 10, pp. 639-642. D0I: https://doi.org/ $10.3103 /$ S1068371217100091

4. Jose A.R. Sistemas de ingenieria. La Habana, Editorial Félix Varela, 2000. $482 \mathrm{p}$.

5. Iliana G. P. Optimization of reactive power compensation in industrial power supply networks using a multi criteria algorithm. Energy, 2006, vol. XXVII, no. 2, pp. 40-44.

6. Alejandro C., Demetrio R.L., Juan M. Metaheuristic optimization applied to the calculation of optimum load flow in electric power grids with mixed type control variables. Engineering Journal UC, December 2013, vol. 20, no. 3, pp. 71-78. D0I: http//www.redalyc.org/artuculo.oa?id: 70732641009

7. Iliana G.P., Secundino M.R. Reactive power compensation by evolutionary techniques. The VII International Conference for the Exploitation of Mineral Resources «CINAREM 2013». Moa, province of Holguín, Cuba, November 19-21, 2013. pp. 1-7.

8. Yun Wei Li, Jinwei He. Distribution system harmonic compensation methods: an Overview of DG-Interfacing inverters. IEEE. Industrial Electronics Magazine, November 2014, no. 8, Iss. 4, pp. 18-31. DOI: 10.1109/MIE.2013.2295421

9. Chkliarsky Y.E., Gonsales P.I. Reactive power compensation optimization and its in the electrical network complex. Journal of Mining Institute, 2011, vol. 194, pp. 349-352. In Rus.

10. Iliana G.P., Secundino M.R., Aristides L.L., Daniel M.E. Metaheuristics based on the variables integration method applied to reactive power compensation in multi-objective optimization. Proc. of the International Conference on Applied Mathematics, Computational Science \& Engineering (AMCSE 2015). Crete, Greece, October 17-19 2015. pp. 207-212.

11. Gonsales P.Z. Selection of parameters economically advantage reactive power compensation. Journal of Mining Institute, 2011, vol. 194, pp. 235-239. In Rus.

12. Yutao Qi, Qingsai Zhanga, Xiaoliang Ma, Yining Quana, Qiguang Miaoa. Utopian point based decomposition for multi-objective optimization problems with complicated Pareto fronts. Applied Soft Computing, December 2017, vol. 61, pp. 844-859. D0I: https://doi.org/10.1016/j.asoc.2017.08.036

13. Abd-Elazim S.M., Ali E.S. Synergy of particle swarm optimization and bacterial foraging for TCSC damping controller design.
Journal of WSEAS Transactions on Power systems, April 2013, vol. 8, no. 2, pp. 74-84.

14. Ali E.S., Abd-Elazim S.M. Power system stability enhancement via bacteria foraging optimization algorithm. Arabian journal for Science and Engineering (AJSE), March 2013, vol. 38, no. 3, pp. 599-611. DOI: $10.1007 / \mathrm{s} 13369-012-0423-\mathrm{y}$

15. Ali E.S., Abd-Elazim S.M. Optimal PSS design in a multimachine power system via bacteria foraging optimization algorithm. Journal of WSEAS Transactions on Power systems, October 2013, vol. 8, no. 4, pp. 186-196.

16. Bedoya-Puerta J.A., Hernandez-Orallo J., Ferri C., RamrezQuintana M.-J. Applying distances between terms to both at and hierarchical data. Proc. of AAIP 2011. $4^{\text {th }}$ International Workshop on Approaches and Applications of Inductive Programming. Denmark, July 2011. pp. 1-16.

17. Gonzalez P.I., Marrero R.S., Legra L.A., Leon S.A., Proano M.X. Modeling of electric network regimes in the presence of harmonic distortion. Energy Technical Magazine, 2017, no. 13, pp. 97-105.

18. Barrico C., Antunes C.H., Pires D.F. Robustness analysis in evolutionary multi-objective optimization applied to VAR planning in electrical distribution networks. Evolutionary Computation in Combinatorial Optimization. EvoCOP 2009. Lecture Notes in Computer Science. Eds. C. Cotta, P. Cowling. Berlin, Heidelberg, Springer, 2009. Vol. 5482, pp. 216-227. DOI: https://doi.org/10.1007/978-3-642-01009-5_19

19. Muthukumar K., Jayalalitha S. Multiobjective hybrid evolutionary approach for optimal planning of shunt capacitors in radial distribution systems with load models. Ain Shams Eng J, 2017, pp. 1-14. DOI: http://dx.doi.org/10.1016/j.asej.2017.02.002

20. Vasconcellos D.B., Abril I.P., Martínez V.L. Reactive power compensation in unbalanced systems using genetic algorithms. Ingeniare.Chilean journal Engineering, 2012, vol. 20, no. 3, pp. 284-292. DOI: http://dx.doi.org/10.4067/S0718-33052012000300002.

21. Secundino M.R., Iliana G.P., Arístides L.L. Convergence analysis of the variables integration method applied in multiobjetive optimization in the reactive power compensation in the electric nets. DYNA 82 (190). April 2015. pp. 160-165. DOI: http://dx.doi.org/10.15446/dyna.v82n190.43697

22. Sharaf A.M., Elgammal Adel. A novel discrete multiobjective particle swarm optimization (MOPSO) of optimal shunt power filter. IEEE/PES Power Systems Conference and Exposition. 2009. pp. 1-7. DOI: 10.1109/PSCE.2009.4839957

23. Schweickardt G.A. Multiobjective metaheuristic FPSO- $\chi$. An application to $\mathrm{mid} /$ long term expansion planning of an electric distribution system. Energetica, 2009, no. 42, pp. 73-88. D0I: 10.15446/energética

24. Rueda V.M., Velazquez J.D., Franco C.J. Recent advances in load forecasting using nonlinear models. DYNA, 2011, no. 78 (167), pp. $36-43$.

25. Shklyarskiy E., Skamyin A.N. Compensation of the reactive power in the presence of higher voltage harmonics at coke plants. Coke and Chemistry, 2016, vol. 59, no. 4, pp. 163-168. DOI: https://doi.org/10.3103/S1068364X16040062.

Received: 22 December 2017.

\section{Information about the authors}

Iliana Antonia G. Palau, Cand. Sc., professor, Cotopaxi Technical University.

Secundino M. Ramirez, Cand. Sc., dean of the department, Cotopaxi Technical University.

Mikhail S. Balabanov, postgraduate student, Omsk State Technical University.

Aristides L. Lobaina, Cand. Sc., assistant professor, Higher Metallurgical Mining Institute of Moa, Dr. Antonio Nunez Jimenez.

Daniel Mendiola Ellis, Cand. Sc., assistant professor, Higher Metallurgical Mining Institute of Moa, Dr. Antonio Nunez Jimenez. 